\title{
Structural Analysis of a Prototype Fast Shutter for ITER cCXRS Diagnostic
}

\author{
A. Panin, W. Biel, Y. Krasikov, O. Neubauer, and D. A. Castaño Bardawil
}

\begin{abstract}
Optical lifetime of the first mirror is a critical issue for the ITER upper-port-plug core-charge-exchange-spectroscopy diagnostic. A fast shutter is engaged to protect the mirror from depositions between measurements. The prototype shutter will be examined in a test vacuum vessel that is now under development in Forschungszentrum Jülich, Germany. Being located near the plasma, the shutter operates under severe thermal and electromagnetic (EM) loads. The multifield analyses conducted for the shutter are presented in this paper. Since the fast shutter can operate within $0.7 \mathrm{~s}$, its static structural analysis should be accompanied by dynamic studies. This paper gives details about the numerical strategy used for a multifield ANSYS modeling of a complex structure. The shutter structural performance under the service, thermal, and EM loadings is in line with the requirements. A solution for a problem of high local thermostresses revealed by the analysis is proposed. Problems connected with other possible port-plug-shutter layouts are discussed.
\end{abstract}

Index Terms-ITER, multifield analyses, shutter, upper-portplug diagnostic.

\section{INTRODUCTION}

$\mathbf{F}$ ORSCHUNGSZENTRUM JÜLICH (FZJ), Germany, with partners has been developing concepts for the ITER upperport-plug core-charge-exchange-spectroscopy diagnostic system that represents a labyrinth of optical mirrors [1]. The first mirror (M1), the closest to plasma, is the most vulnerable structure component subjected to depositions that drastically reduce its optical performance. To decrease the mirror degradation, a fast shutter is engaged. The operations of the shutter and diagnostic neutron beam (DNB) should be synchronized. The fast shutter can increase the M1 lifetime up to eight times. Located in the near-plasma harsh environment, the shutter experiences high thermal and electromagnetic (EM) loads. An intermediate shutter design option was briefly presented in [2]. This paper deals with the prototype shutter for the FZJ port-plug reference design. The shutter will be examined in a test vacuum vessel that is under development in the FZJ. Multifield analyses have been conducted to evaluate the shutter structural performance.

Manuscript received August 23, 2011; revised November 15, 2011; accepted December 18, 2011. Date of publication February 3, 2012; date of current version March 9, 2012. This work was supported in part by the German Ministry of Research under Grant 03FUS0007 and in part by the European Community under contracts between EURATOM and Forschungszentrum Jülich, TNO, CCFE, and HAS.

The authors are with the IEK4-Plasma Physics, Forschungszentrum Jülich $\mathrm{GmbH}$, Association EURATOM-FZJ, Trilateral Euregio Cluster, D52425 Jülich, Germany (e-mail: a.panin@fz-juelich.de; w.biel@fz-juelich.de; y.krasikov@fz-juelich.de; o.neubauer@fz-juelich.de; d.castano.bardawil@fzjuelich.de).

Color versions of one or more of the figures in this paper are available online at http://ieeexplore.iee.org.

Digital Object Identifier 10.1109/TPS.2012.2182782
The shutter structural features and numerical approaches to build the ANSYS finite element (FE) models [3] for multifield static/dynamic studies are presented in Section II. Sections III-V describe the shutter structural behavior under the pressure load, the shutter EM-structural, and thermostress analyses. The shutter mechanical performance is discussed in Section VI.

\section{Shutter Mechanical Structure AND FE MODELING}

\section{A. Shutter Mechanical Structure}

The steel shutter consists of two flexible arms $\sim 1.5 \mathrm{~m}$ long carrying protective blades at their front ends and attached to a gas actuator at the rear ends (Fig. 1). The arm inner parts are bolted to the movable actuator piston, while the outer ones are bolted to the stationary actuator housing. The actuator is fixed to the retractable tube (RT) that holds the M1 together with the shutter and allows their maintenance. A water cooling tube is welded to the arms in a way to limit the arms' temperature and minimize their thermal distortion. The blades taking the most heat load are directly welded to the loops of the cooling tube.

The passively cooled gas actuator consists of two chambers, i.e., the outer $(\mathrm{P}-)$ and inner $(\mathrm{P}+)$ ones, separated by bellows. Both are initially pressurized. When the pressure in the outer chamber is higher than that in the inner one, the shutter is closed. With the pressure rise in the inner chamber, the piston moves forward, making the arms bend laterally. The blades move apart, thus opening the mirror for measurements. To avoid friction/bearing mechanisms, the movable piston is attached to the housing via sets of elastic flexible disks, guiding the piston longitudinally and resisting loads acting on the shutter arms. To fix the arms in their extreme positions, the outer and inner bumpers are designed. The bumper system includes soft pads and stoppers that face the pads. For stability reason, certain arms' preloading against the bumpers is provided in both shutter extreme positions. The blades, to facilitate a heat transfer to the cooling tube while limiting induced eddy currents, are designed as a set of steel sheets cladding bronze strips. The main requirements to the fast shutter are as follows.

1) The closed shutter should provide maximum protection for the M1 during operation and baking.

2) The open shutter should provide access for a full DNB signal on the mirror in the time range of 1-10 s.

3) The shutter should operate within $0.7 \mathrm{~s}$. Its arms have to keep stable on the bumpers during service and EM loadings.

4) The shutter should satisfy the ITER structural criteria. 


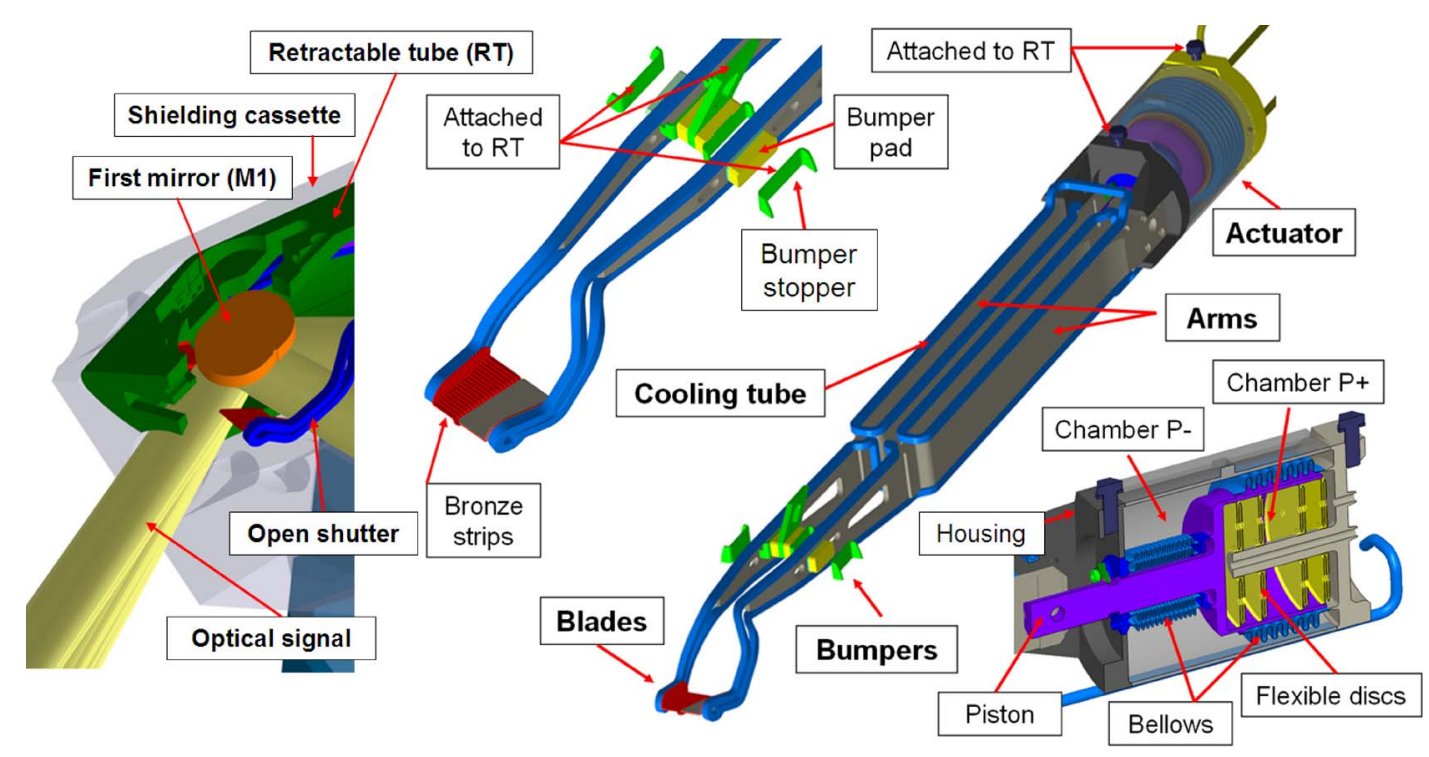

Fig. 1. Details of fast shutter structure.

\section{B. FE Modeling}

Modeling strategy for numerical multifield problems has to be carefully built up.

1) Aim of modeling. The main goals were an evaluation of the shutter static strength and a study of the shutter dynamic movements. The modeling was a compromise between the models' accuracy in high-field-gradient regions and limitation on the model size to allow timeconsuming nonlinear static and transient analyses. The revealed local problems are a subject of a further detailed submodeling.

2) $C A D$ geometry reasonable simplification. Redundant model details like chamfers, fillets, threads, etc., distant from expected field concentrators, are removed. The bolted/keyed connections are modeled as surface-tosurface contacts. Loads on the bolts/keys are calculated from the nodal reaction forces.

3) FE model flexibility. Since the main shutter parts, like the arms, cooling tube, blades, and elements of the actuator, underwent periodical design changes, they were meshed separately and connected via their interfaces. It is convenient to develop the FE models of some parts, like the cooling tube in this analysis, in a parametric way.

4) Part merging. The ANSYS offers different tools to link parts with dissimilar meshes (contact "bonded always" option and constraint equations). A key feature of the shutter structural behavior is a bending of the relatively thin arm plates together with the welded cooling tube. Since the tube contributes up to $75 \%$ of the arm bending stiffness, the structure is very sensitive to a contact algorithm used to merge these parts and to transfer the shear forces from the arm plate to the tube. The ANSYS MPC and pure Lagrange algorithms, based on the Lagrangemultiplier method, proved to work correctly. Other algorithms, like penalty, depending on the stiffness of the artificial interface springs, are not quite reliable for such problems.
5) Choice of an FE type. A tradeoff between the linearversus-quadratic and hexahedral-versus-tetrahedral element types was an important modeling issue. Combination of the linear elements with the more accurate quadratic ones was used in the thermostructural analyses (see Section III). The ANSYS EM models are restricted to the linear elements only. The hexahedral elements (linear or quadratic) were mostly used for all analyses. Note that meshing with these more precise elements requires certain effort compared to free meshing.

6) Load transfer. For the thermal-structural models, which utilize the same meshes, a direct nodal force (temperature) transfer was possible. The EM model is built with the linear elements only. The corner nodes of the structural quadratic elements were used to build the EM linear ones in the same locations. The EM nodal forces were directly transferred to the corner nodes of the structural elements, including the quadratic ones. Despite the different element shape functions, this simple engineering method was proved to provide the correct summed moments on the structure parts because of the relatively small element size. To transfer the EM forces to the structural dynamic model, having more rough mesh, the external transfer code was employed. It seeks for each "loaded" node of the source model (EM) the geometrically nearest nodes of the target (structural) model. The method implies engineering considerations of a force distribution, a sound choice of an epsilon neighborhood for target nodes, and reliable checks of calculated moments.

\section{Structural Analysis of Shutter Under SERVICE PRESSURE LOAD}

\section{A. Structural FE Modeling}

Most of the structure is meshed with the linear hexahedral elements. The thin bellows and flexible disks, experiencing the highest bending, are modeled with the quadratic elements. The 


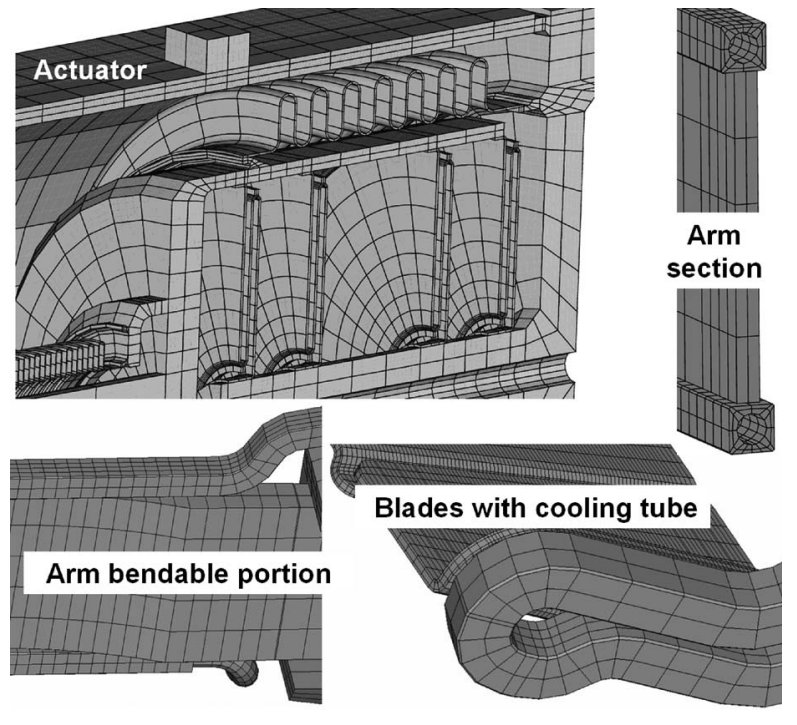

Fig. 2. Shutter meshing details.

arms' bendable parts, being the expected locations of the stress gradients, have a reasonably fine mesh. The blades are also meshed relatively fine, keeping in mind the following EM and thermal analyses (Fig. 2).

Separately meshed model parts are linked together. A nonlinear contact behavior is modeled between the arms and bumpers. The actuator, as well as the inner bumper pads and outer bumper stoppers, is held by the RT. Since the RT is not modeled, these elements are numerically fixed in space. The FE model, counting up to 125000 elements and resulting in 835000 equations, looks well balanced in terms of model size and the multifields' expected accuracy.

The material properties (316LN-IG and $\mathrm{CuCrZr-IG)}$ are specified according to [4]. The linear elastic soft foam material ( $10 \%$ steel) is assumed as a pad material. A proper pad material will be further selected according to [5]. The allowable stresses for structural materials are defined according to [6]. The loading history includes the gravity and actuator pressures to preload the closed shutter $(P-=0.08 \mathrm{MPa} ; P+=0.04 \mathrm{MPa})$ and to open and preload the open shutter $(P-=0.08 \mathrm{MPa} ; P+=$ $0.352 \mathrm{MPa}$ ). The shutter working temperature is assumed to be $70{ }^{\circ} \mathrm{C}($ Section V).

\section{B. Shutter Under Pressure Load: Static Analysis}

The chosen pressure force to preload the closed shutter is $0.5 \mathrm{kN}$. To open the shutter, a force of $3.9 \mathrm{kN}$ is required, and an additional force of $0.8 \mathrm{kN}$ was chosen to preload the open shutter. When the shutter is open, its arms are bent apart by $40 \mathrm{~mm}$ each, and the actuator piston stroke is $1.9 \mathrm{~mm}$ (Fig. 3).

No problems were found in the blade bronze strips. The stresses in the open shutter are given in Table I and Fig. 4. The bending stresses prevail. The stresses satisfy the ITER Level A structural criteria for the loading conditions of Category I [7]. Stresses in the arms and cooling tube are modest. The large bellows and flexible disks experience rather high bending, mostly caused by their axial movement during shutter opening coupled with the pressure load. The von Mises elastic stain

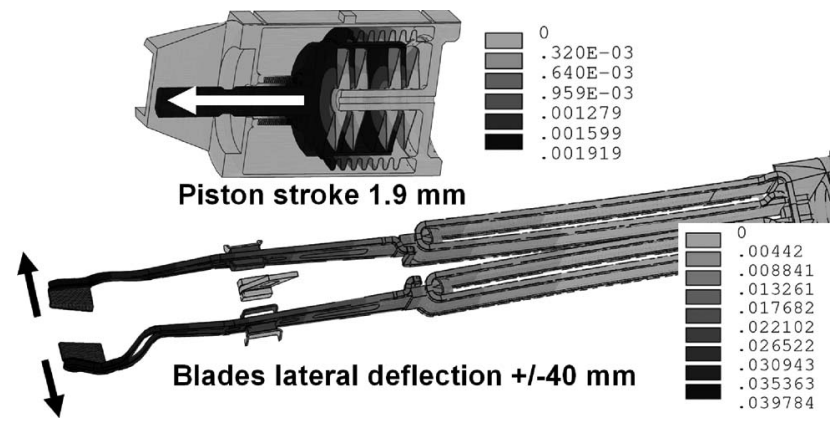

Fig. 3. Open shutter: Piston and arm movements.

TABLE I

Open Shutter. CAlculated Primary Stress Intensities Versus Allowable Stresses in STEEL Components

\begin{tabular}{|c|c|c|}
\hline & Membrane, $\mathrm{MPa}$ & Membrane + bending, MPa \\
\hline Arm & $10 / 147$ & $<96 / 220$ \\
\hline Cooling tube & $6 / 147$ & $91 / 220$ \\
\hline Flexible disc & $<30 / 147$ & $165 / 220$ \\
\hline Bellows & $<40 / 147$ & $186 / 220$ \\
\hline
\end{tabular}

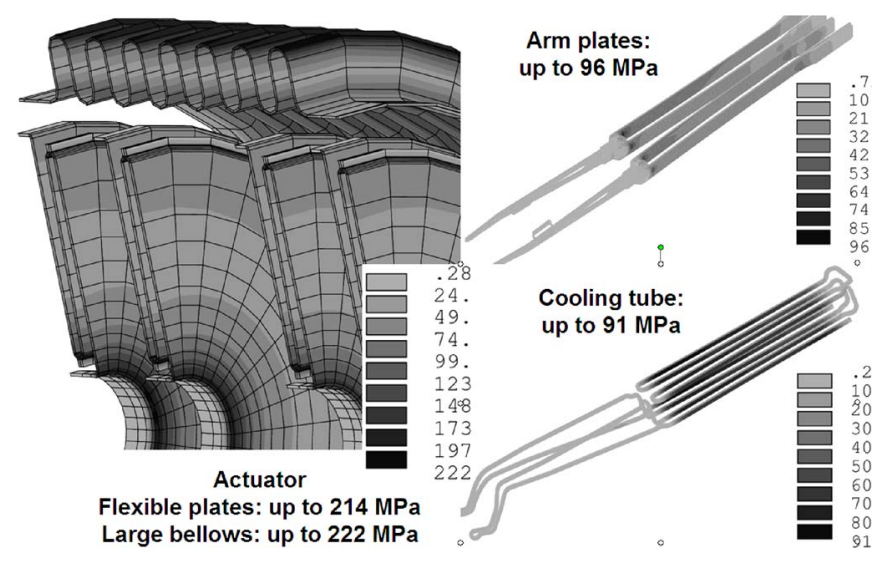

Fig. 4. Tresca stress intensity distribution in open shutter (in megapascals).

range of $0.11 \%$, found in the flexible disks, is used for fatigue evaluation (Section VI).

An important safety feature of the shutter is that, in both extreme positions, its arms are locked by the bumpers against further bending in case of some accidental pressure rise. The axial stopper limiting the piston stroke is also foreseen.

\section{Shutter Under Pressure Load: Dynamic Analysis}

Since the shutter has to be fully open after $0.7 \mathrm{~s}$, its dynamic oscillations should be studied. The shutter movable portion includes two arms ( $1.5 \mathrm{~m}$ long and $13 \mathrm{~kg}$ each) and the actuator piston $(5.4 \mathrm{~kg})$ bolted to the arms. To preload the open shutter, the initial pressure in the outer actuator chamber is chosen higher than that in the inner one. When the pressure in the inner chamber exceeds that in the outer one, the arms open until they touch the outer bumpers. The pressure still rises to preload the arms on the bumpers and to suppress main rebounds of the arms. The arms' free ends, extending $\sim 0.4 \mathrm{~m}$ above the bumpers, oscillate in the bending mode while the piston oscillates axially. The structure exhibits similar behavior during its closing. The modal analysis of the structure was done first. The 


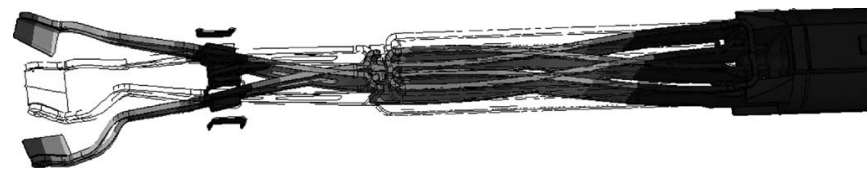

Fig. 5. Modal analysis. Shutter oscillating around bumpers $(25 \mathrm{~Hz})$.
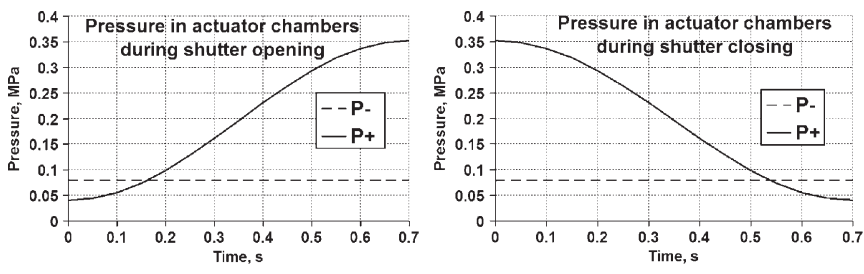

Fig. 6. Pressure in actuator chambers to open and close the shutter.

first natural frequency of the free bending shutter (no contact with bumpers) is $5.5 \mathrm{~Hz}$. The main modes, corresponding to the shutter arms' oscillations around the bumpers, have frequencies of 25 and $45 \mathrm{~Hz}$ (Fig. 5).

The ANSYS full transient dynamic analysis was used. The sinusoidal pressure rise/drop in the inner chamber during $0.7 \mathrm{~s}$ was modeled (Fig. 6). To resolve the dynamic response of the structure up to a frequency of $\sim 400 \mathrm{~Hz}$, an integration time step of $80 \mu \mathrm{s}$ was chosen. Note that the structure response frequencies up to $\sim 300 \mathrm{~Hz}$ were revealed by the analysis during rebounds. The expected sources of energy dissipation in the shutter, working in vacuum, are internal material damping (likely in long welds between the arms and cooling tube), friction in the bolted-arm-actuator connections, and heating of the actuator gas due to the piston movement. The bumper pads (metal foam) are assumed to work in vacuum as linear elastic elements and do not absorb energy. The ITER load specification for seismic events gives, for the welded steel or bolted steel with friction connection, a damping factor of $3 \%$ of the critical damping for all modes [7]. In the analysis, a Rayleigh stiffness matrix multiplier of $8 \times 10^{-5}$ was assumed for the entire structure. For the main expected oscillation modes (25-40 Hz), the damping ratio varies from $0.6 \%$ to $1 \%$ of the critical damping. To decrease computational time, more rough FE mesh is used for this analysis compared to the static one.

Since the bumpers restrain the arms' movements, the structure dynamic stress state has not visibly changed compared to the static solution. The issue is the arms' oscillations, which can deteriorate the shutter performance. Just before the impact, the arms' velocity is less than $0.18 \mathrm{~m} / \mathrm{s}$, and the shutter kinetic energy is modest (under $33 \mathrm{~mJ}$ ). High amplitude rebounds decay within $0.2-0.3 \mathrm{~s}$, during preloading of the open or closed shutter (Fig. 7). Moderate amplitude oscillations $( \pm 0.6 \mathrm{~mm})$ with frequencies of $25-40 \mathrm{~Hz}$ do not deteriorate the mirror performance. Note that the blades of the closed shutter initially overlap by $\sim 1.5 \mathrm{~mm}$. Thus, during service, the shutter is ready to measurement or fully closed after $0.7 \mathrm{~s}$. The calculated average and peak pressures on the bumper pads will serve to select a proper pad material. It is desirable that the pads could be plastically deformed after the first loading cycles and their contact with the arms (not optimal due to the shutter assembly tolerances) could be "adjusted." Real oscillations will

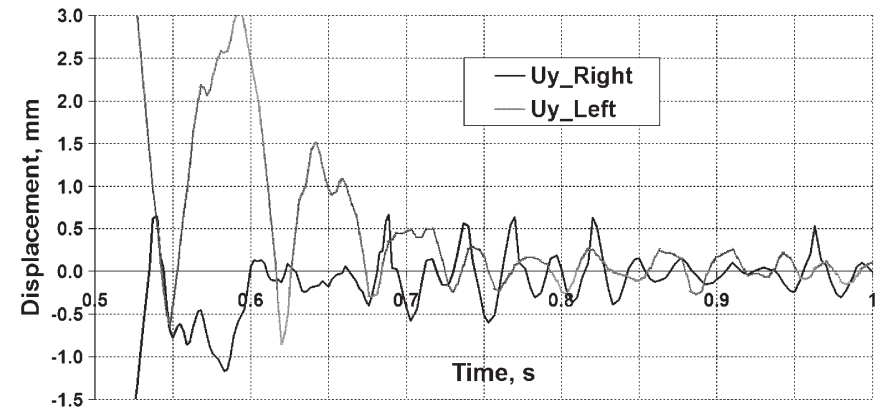

Fig. 7. Lateral oscillations of blades during shutter closing.

be monitored during testing of the prototype shutter in the vacuum chamber.

\section{Shutter EM-Structural Analyses}

\section{A. Shutter EM Analysis}

The shutter EM-structural modeling utilizes the "express" approach. For clarity, although this method was described and validated for shutter models in [2], its main features are shortly summarized as follows.

For small conducting components, hosted by massive shielding structures, their detailed EM models can be built without "air" elements connecting the conducting parts. The boundary conditions for such models are taken from the global EM model containing the massive structures only. The impact of the eddy currents, induced in a small component, on the changing external magnetic flux is neglected.

The global ITER EM model addressing the main upper-portplug features was developed and validated by FZJ [2]. The electrical connections between the plug structures do not let the halo current to flow through the shutter. The fast linear plasma VDE scenario (36 ms) was found to result in the highest plug eddy currents. This scenario was selected for the shutter EM analysis. The fast VDE falls into the loading Category II [7].

The boundary conditions on the outer surfaces of the shutter conducting elements (components of the vector potential) are derived from the global EM model. To connect dissimilar meshes (by VOLT degrees of freedom), the ANSYS Coupling Adjacent Regions method was used. To avoid high bending moments on the shutter due to the eddy currents, bridging the shutter with the RT via the bumpers and actuator, the bumpers are electrically isolated.

The transient EM analysis has been performed for both extreme shutter configurations: open and closed. No visible difference in the results for closed and open shutters was found. The mechanical moments on the shutter arms peak at the end of the fast VDE (Fig. 8). Each arm is mainly loaded by a twisting moment of $\sim 45 \mathrm{~N} \cdot \mathrm{m}$. These moments are determined by the eddy current loops closing in the arm plates. The currents flowing in the blades contribute to a lesser extent.

\section{B. Shutter Under EM Loading: Static/Dynamic Stress Analyses}

The EM forces corresponding to the maximal mechanical moments on the shutter were used for the static stress analysis. 


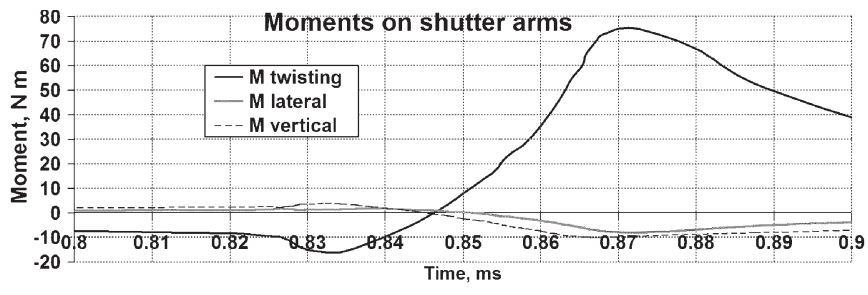

Fig. 8. Mechanical moments on the closed shutter due to EM loading.

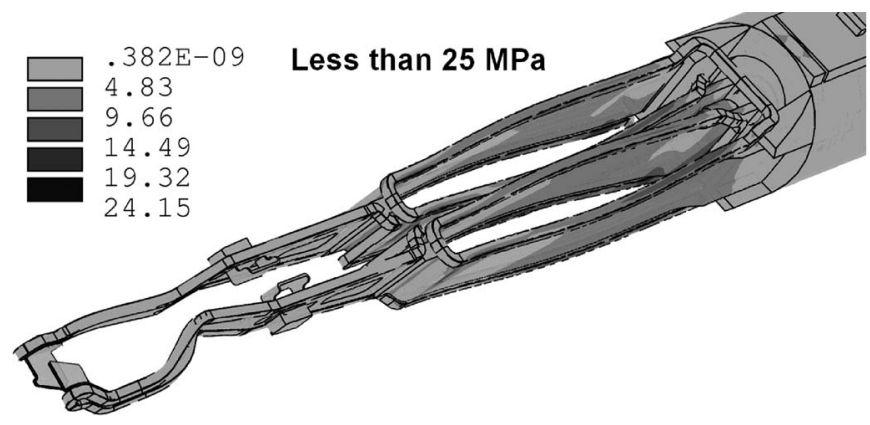

Fig. 9. Stress intensity distribution in open shutter (in megapascals) under EM loading only. Stresses due to shutter opening are subtracted.

TABLE II

Open Shutter Under EM Loading. Calculated Primary Stress InTENSITIES Versus ALLOWABLE STRESSES IN STEEL COMPONENTS

\begin{tabular}{|c|c|c|}
\hline & Membrane, MPa & Membrane + bending, MPa \\
\hline Arm & $10 / 147$ & $<115 / 220$ \\
\hline Cooling tube & $6 / 147$ & $106 / 220$ \\
\hline Flexible disc & $<30 / 147$ & $173 / 220$ \\
\hline Bellows & $<40 / 147$ & $186 / 220$ \\
\hline
\end{tabular}

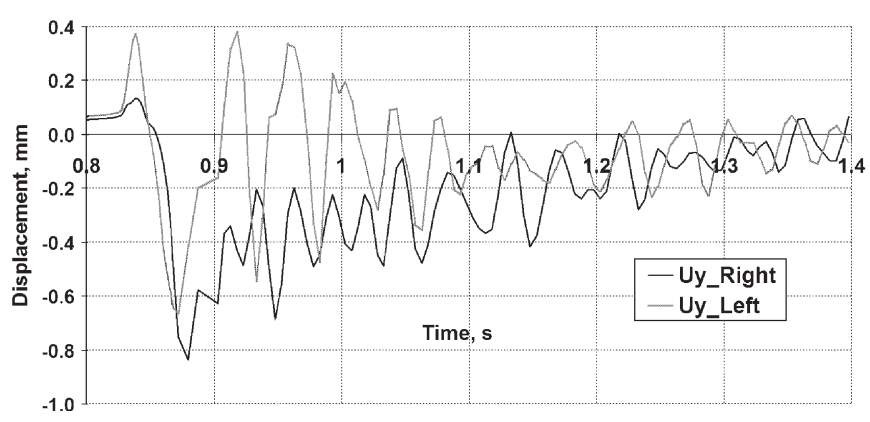

Fig. 10. Lateral oscillations of blades of closed shutter under EM loading.

The impact of the EM loads on the stress state of the open shutter is relatively small (Fig. 9). Additional bending of the cooling tube due to the arms' twisting was revealed. The twisting of the inner arms is resisted by the flexible disks. Table II summarizes the main calculation results. The stresses in the open shutter under the EM forces are within the static allowable limits [7]. The von Mises elastic stain range of about $0.115 \%$, found in the flexible disks and large bellows, is used for fatigue evaluation (Section VI).

For the dynamic analyses of the closed shutter, the whole loading time history was employed. The oscillations around the bumpers start at the end of the VDE, and the high amplitude movements decay within $0.3 \mathrm{~s}$ (Fig. 10). Even during oscillations, the blades of the closed shutter still overlap and can prevent the M1 from depositions.

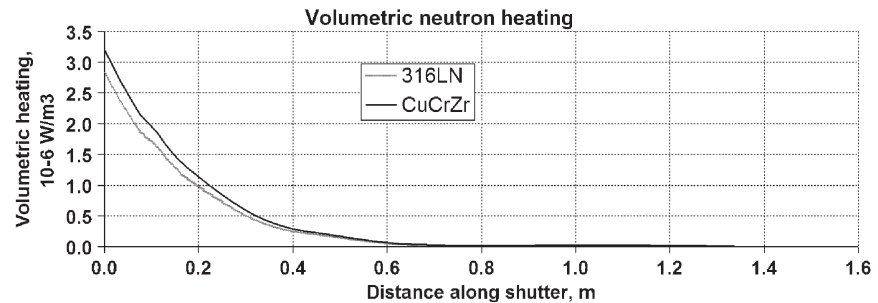

Fig. 11. Volumetric neutron heating along the shutter (from the plasma side).

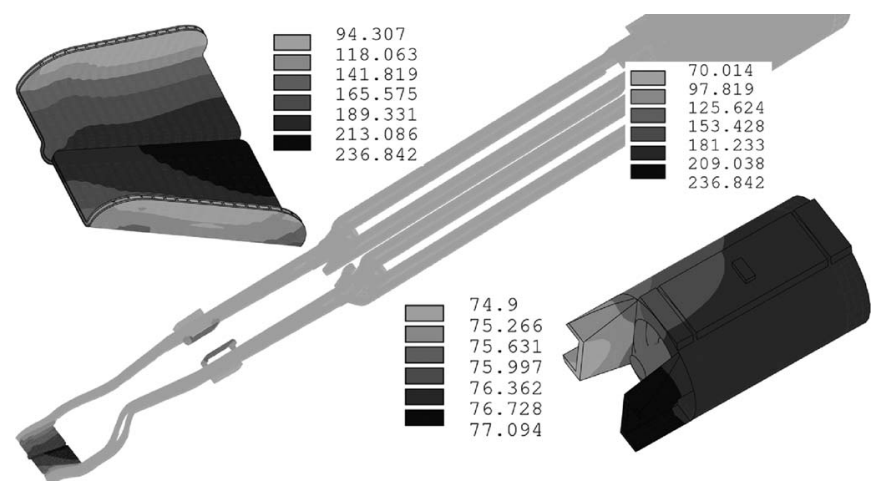

Fig. 12. Temperature field in shutter (in degrees Celsius). The water flow rate is $0.3 \mathrm{~m} / \mathrm{s}$.

\section{Shutter Thermostress Analysis}

\section{A. Shutter Steady-State Thermal Analysis}

The structure is mainly heated by the volumetric neutron loading. The calculation technique is described in [8], and the recent results for the shutter are plotted in Fig. 11. The shutter blades are additionally subjected to radiation heat fluxes coming from the plasma and M1. They are estimated as 20 and $4 \mathrm{~kW} / \mathrm{m}^{2}$, respectively. The total thermal power absorbed by the structure is $670 \mathrm{~W}$. The third of this power is due to the radiation fluxes. The actuator, distant from plasma, absorbs only $0.3 \mathrm{~W}$.

The shutter arms are actively cooled by the water flowing through the cooling tube. The gas $(\mathrm{He})$, pressurizing the actuator, was initially aimed for its passive cooling. Because of low thermal power coming to the actuator, the actuator is assumed to be only conductively cooled via the arm-piston contacts. This can simplify the layout of a gas supply system.

The main water parameters used for calculation are a tube hydraulic diameter of $-8 \mathrm{~mm}$, an inlet temperature of $-70^{\circ} \mathrm{C}$, a pressure of $-3 \mathrm{MPa}$, a flow rate of $-0.3 \mathrm{~m} / \mathrm{s}$, and a heat transfer coefficient of $-7900 \mathrm{~W} / \mathrm{m}^{2} \cdot \mathrm{K}$ (turbulent flow).

An ideal contact (no contact resistance) was used to link the parts together (contact technology). Only on the interfaces, where the arms are bolted to the actuator, a contact conductance of $500 \mathrm{~W} / \mathrm{m}^{2} \cdot \mathrm{K}$ was specified. It was estimated according to [9], and a quite conservative value was assumed for the analysis. The same FE mesh as for the structural analysis was used for the thermal one. The calculated temperature field is shown in Fig. 12. The temperature rise in the blades achieves $167 \mathrm{~K}$. The temperature change over the actuator is $2 \mathrm{~K}$. The heating of the cooling water is $11 \mathrm{~K}$. 

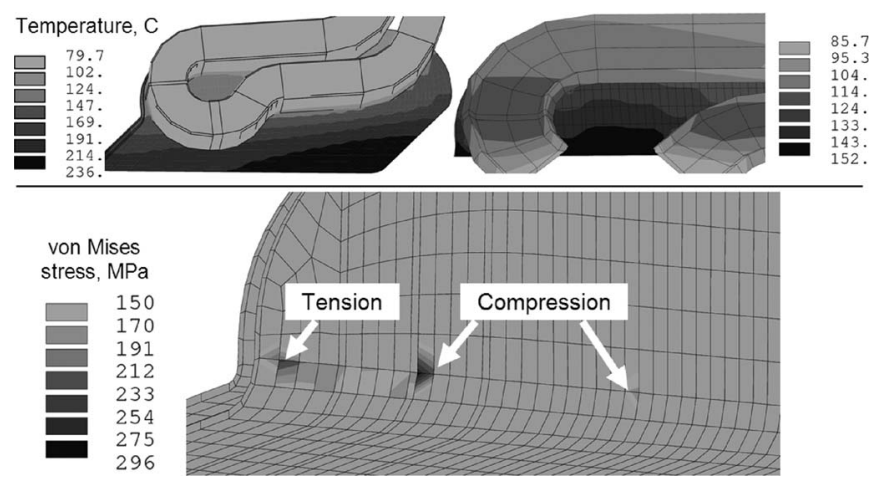

Fig. 13. Temperature field and peak elastic thermostress in blades welded to the cooling tube (gray—-for stress under the yield limit at working temperature).

\section{B. Shutter Static Thermostress Analysis}

The nodal temperatures are directly applied to the structural model. Temperature-dependent material properties are used [4]. Slight distortion of the arms, mostly due to the temperature change across the actuator, was found. In both extreme positions, the shutter under the thermal load remains preloaded, and the blades of the closed shutter still overlap.

The thermostresses are categorized as the secondary ones and have no impact on the primary stresses [6]. The stress state in the most loaded components like the flexible disks and bellows did not visibly change due to the thermal loading. The secondary thermostress in the arms and cooling tube is within the allowable limits. The main problem revealed by the analysis is the high thermostresses in the blades.

The problem areas are located at discontinuity between the welded hot blades and cooling tube. The elastically calculated thermostresses on the blade surface along the weld perimeter are shown in Fig. 13 (bottom). The high-temperature gradients in this region can be also seen in Fig. 13 (top). Because of a thermal expansion of the blade heated area, which is clamped inside the loop of the cooling tube, this area is compressed. On the contrary, at the blade unsupported edge, tension develops.

The situation can be improved by using the supporting fillet weld along the discontinuity. The weld can smooth peaks and redistribute temperature and stresses. Further submodeling of these regions is required to address true values of peak thermostresses for fatigue evaluation.

\section{Result Discussion}

The prototype shutter for the FZJ port-plug reference design option has been numerically studied. The main modeling goals are achieved. The static strength of the shutter under the service, EM, and thermal loadings has been confirmed for the ITER structural criteria Level A. The earthquake loading conditions specified by ITER (structural criteria Level C) do not seem to be a problem and can be simulated soon. The buckling analysis will be performed for the small bellows working under an external pressure that is not that high. The shutter baking procedure is out of the scope of this paper.

The model satisfactorily addresses the peak stress in most loaded components for fatigue estimation. The elastic strain range in the flexible disks reaches $0.115 \%$. Even with correc- tions for small plasticity, the strain range is under $0.174 \%$, which is an allowable value for the $316 \mathrm{LN}-\mathrm{IG}$ steel for $10^{6}$ loading cycles [4]. The problem of high cyclic thermostresses in the blades should be solved. The proposed design solution can be studied by means of further submodeling.

The shutter dynamic behavior seems to be in line with the requirements. Using the calculated pressures on the bumper pads, a foam material for the pads can be selected according to [5]. The real oscillations due to the shutter service will be monitored with a fast camera during the prototype testing.

The shutter cooling structure is able to withstand neutron loads specified by the ITER project. For the FZJ port-plug reference design, the shutter actuator can be cooled via conduction to arms, without being passively cooled by a gas. For other plug designs, the considerable radiation fluxes could be an issue for the shutter blades. The matter is under study. Neutron damage of the shutter structural materials is to be taken into account for the shutter options located closer to plasma.

\section{ACKNOWLEDGMENT}

The views and opinions expressed herein do not necessarily reflect those of the European Commission.

\section{REFERENCES}

[1] Y. Krasikov, T. Baross, W. Biel, A. Litnovsky, N. Hawkes, G. Kiss, J. F. F. Klinkhamer, J. F. Koning, and A. Krimmer, "Development of design options for the port plug components of the ITER core CXRS diagnostic," Fusion Eng. Des., vol. 86, no. 9-11, pp. 2055-2059, Oct. 2011, DOI: 10.1016/j.fusengdes.2011.01.086.

[2] A. Panin, W. Biel, Y. Krasikov, and O. Neubauer, "Electromagnetic modeling and subsequent structural analysis for ITER core CXRS upper port plug diagnostic structure," Fusion Eng. Des., vol. 86, no. 9-11, pp. 2016-2020, Oct. 2011, DOI: 10.1016/j.fusengdes.2010.12.030.

[3] ANSYS, Inc., Release 12.0.1.

[4] Structural design criteria for ITER in-vessel components (SDC-IC). Appendix A. Materials design limit data ITER_D_222RLN v2.0.

[5] [Online]. Available: www.ergaerospace.com/Energy-Absorbtion.html

[6] Structural design criteria for ITER in-vessel components (SDC-IC), ITER_D_222RHC v2.0.

[7] ITER load specifications (LS), ITER_D_222QGL v3.5.

[8] P. Bourauel, R. Nabbi, W. Biel, and R. Forrest, "A full automatical coupling method for Monte Carlo code MCNP and activation code FISPACT with high-dimensional visualization capabilities," Jahrestagung Kerntechnik, Dresden, Germany, 2009

[9] Y. Mesnyankin, A. G. Vikulov, and D. G. Vikulov, "Solid-solid thermal contact problems: Current understanding," Physics-Uspekhi, vol. 52, no. 9, pp. 891-914, 2009, DOI: 10.3367/UF Ne.0179.200909c.0945.

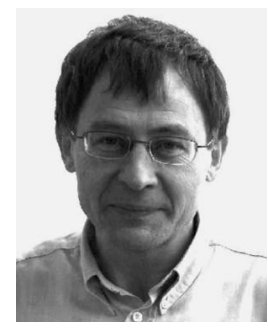

A. Panin received the Diploma of EngineerPhysicist (M.S. degree) in dynamics and strength of machines from St.-Petersburg State Polytechnical University, St.-Petersburg, Russia, in 1979.

He was with the D.V. Efremov Institute, St.-Petersburg, Russia, until 2004, where he was a Member of the ITER Russian Home Team. He is currently a Research Scientist with Forschungszentrum Jülich, Jülich, Germany. He is mostly focused on a multifield analysis for unique fusion and electrophysical devices. 


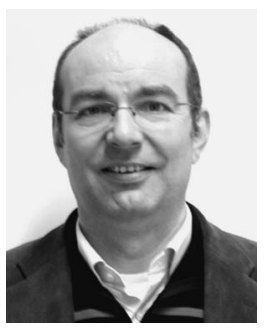

W. Biel received the Ph.D. degree in plasma physics from Heinrich-Heine-Universität Düsseldorf, Düsseldorf, Germany, in 1996.

Since 1997, he has been a Research Scientist with Forschungszentrum Jülich, Jülich, Germany, where he is currently the Project Leader for the development of the ITER CXRS diagnostic system. The main field of his work is the development of plasma diagnostics.

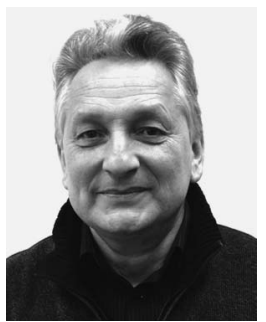

Y. Krasikov received the M.S. degree in mechanical engineering from St.-Petersburg State Polytechnical University, Saint Petersburg, Russia, in 1983.

He was the Design Group Leader with the D. V. Efremov Institute, Saint Petersburg, Russia, until 2004. He is currently the Head of the Design Group, Forschungszentrum Jülich, Jülich, Germany.

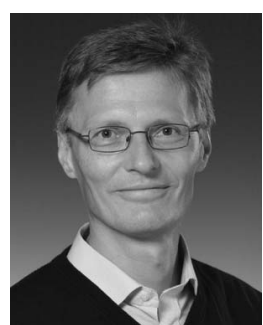

O. Neubauer received the M.S. degree in electrical engineering and the Ph.D. degree from Dresden University of Technology, Dresden, Germany, in 1987 and 1991, respectively.

$\mathrm{He}$ is currently the Head of Fusion Technology with the Institute of Energy and Climate ResearchPlasma Physics, Forschungszentrum Jülich, Jülich, Germany. His responsibilities include the management of projects in engineering science.

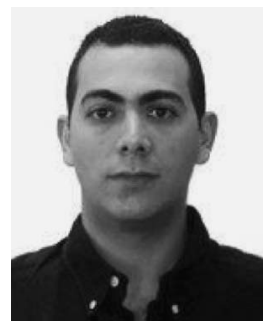

D. A. Castaño Bardawil received the B.S. degree in mechanical engineering from the Technological University of Pereira, Pereira, Colombia, in 2006 and the M.S. degree in energy systems from Aachen University of Applied Sciences, Aachen, Germany, in 2010 .

He is currently with Forschungszentrum Jülich, Jülich, Germany. 